\title{
Strukturwandel und therapeutische Interaktion im Krankenhaus
}

\author{
Friedrich Heubel • Matthias Kettner · Arne Manzeschke
}

Online publiziert: 18. März 2012

(C) Springer-Verlag 2012

Der weltweit zu beobachtende Strukturwandel des Gesundheitswesens, nämlich der Trend zur Großräumigkeit, zu industrieartigen Versorgungsformen und zur Konzeptionalisierung als Wirtschaftsfaktor, wird üblicherweise als Ökonomisierung oder Kommerzialisierung identifiziert. Im Krankenhaus besteht er in einer Tendenz zur strukturellen Verselbständigung des Managements. Sie reicht von mehreren Stufen der formellen bis zur materiellen Privatisierung, d.h. dem Übergang aus öffentlicher in private Trägerschaft. Der so verstandene Strukturwandel begegnet aber sowohl in der Öffentlichkeit wie den Gesundheitsberufen erheblichen Vorbehalten. Die Arbeitsgruppe Ökonomisierung in der Akademie für Ethik in der Medizin ist - anlässlich der Privatisierung eines Universitätsklinikums - diesen Vorbehalten nachgegangen [1]. Wir haben den so beschriebenen Strukturwandel in eine ethische Perspektive gestellt und gefunden, dass die weitverbreitete Intuition, privates Gewinninteresse vertrage sich nicht mit dem therapeutischen Auftrag von Krankenhäusern, einen rational aufweisbaren Kern hat. Die Entlassung der Institution Krankenhaus aus öffentlicher Verantwortung stellt einen moralisch relevanten Defekt dar. Die abschließende Stellungnahme in unserem 2010 erschienenen Buch nennt die Privatisierung von Krankenhäusern moralisch problematisch, solange nicht institutionell gesichert ist, dass zwischen dem medizinisch Angezeigten und dem finanziellen Gewinninteresse auf transparente Weise abgewogen wird.

An diese Überlegungen schließen wir mit dem vorliegenden Schwerpunktheft an. In der Bewertung als moralisch problematisch ist nämlich eine nichtadressierte Handlungsaufforderung enthalten: Der als moralisch problematisch bewertete Zustand „sollte“ zu einem

PD Dr. med. F. Heubel $(\bowtie)$

Im Stiftfeld 17, 35037 Marburg, Deutschland

E-Mail: heubelfr@staff.uni-marburg.de

Prof. Dr. phil. Dipl. Psych. M. Kettner

Universität Witten/Herdecke, Witten, Deutschland

PD Dr. theol. habil. A. Manzeschke

Institut Technik Theologie Naturwissenschaften,

Ludwig-Maximilians-Universität München, München, Deutschland 
moralisch unproblematischen werden, „man“ sollte oder „man“ müsste ihn korrigieren. Dieser Adressierung wendet sich die Arbeitsgruppe nun zu. Sie fragt nach den Akteuren, von denen ein korrigierendes Handeln erwartet werden darf, und nach ihren Handlungsmöglichkeiten. Dazu ist das Konzept „Organisation“ ein geeignetes Mittel. Denn wenn eine Organisation das Arrangement von geeigneten, einander bedingenden Mitteln ist, und zu diesen Mitteln, wie im Gesundheitswesen, Akteure gehören, dann muss der Organisationszweck mit den Handlungszwecken dieser Akteure abgeglichen sein. Dieser Abgleich von Zwecken ist Sache der organisatorisch Verantwortlichen. Da aber das Organisieren ein Handeln ist, lässt sich auch das Organisieren - nicht nur die vorfindliche Organisation - moralisch bewerten. Wir schließen also jetzt in die ethische Perspektive nicht nur die Organisation, sondern auch die Organisierten und die Organisatoren als Akteure ein. Das Ergebnis kann freilich kein Aktionsplan sein, sondern bewegt sich zwischen der moralisch geleiteten Priorisierung von Zwecken, der Einschätzung von Realisierungsbedingungen und der Verantwortungszuschreibung. Wir sehen die therapeutische Interaktion als den organisatorisch zu bewahrenden Kern der Aufgaben eines Krankenhauses an und weisen der ärztlichen und der pflegerischen Profession dabei eine hervorgehobene Rolle zu.

Horst Imdahl beschreibt die der Betriebswirtschaftslehre entnommenen organisatorischen Maßnahmen, die sich, ausgehend von den Krankenhäusern in privater Trägerschaft, im Interesse der Gewinnsteigerung derzeit ausbreiten. Wegen der durch das DRG-System erzwungenen Konkurrenzsituation kann sich kein Haus diesem Druck entziehen, er dient aber per se nicht der Verbesserung der Versorgung. - Franziska Prütz analysiert den Qualitätsbegriff. Nach den maßgeblichen Definitionen ist Qualität das Ausmaß, in dem sich eine messbare Größe einer objektiven Vorgabe annähert. Das heißt, dass es eine ganze Reihe von objektiv-sachlichen Qualitätsbegriffen gibt, deren Menge aber allenfalls eine Annäherung an das Ideal guter und gerechter Krankenbehandlung liefert. Der Qualitätsdiskussion muss bewusst bleiben, dass auch die „subjektive“ Qualität in der therapeutischen Interaktion ihren gesicherten Raum braucht. - Arne Manzeschke fragt, ob das Konzept von Corporate Governance, das sich gegenwärtig auch im Krankenhaussektor ausbreitet, nicht dessen ,genuines Handlungsziel" konterkariert. Der hohe moralische Anspruch dieses Konzepts steht im Widerspruch zu der faktischen Unklarheit darüber, was ,gute Unternehmensführung“ in der spezifischen Organisation Krankenhaus heißt. Ein spezifischer Kodex würde möglicherweise die adäquate Sachzielorientierung an der therapeutischen Interaktion gewinnen und umsetzen. - Friedrich Heubel schlägt vor, den Schutz der therapeutischen Interaktion als normative Vorgabe sowohl für den Krankenhausträger als auch für eine sich selbst organisierende Profession anzusehen, und demgemäß Handlungsanreize, die professionsspezifische Verantwortlichkeiten bedrohen, organisatorisch auszuschließen. - Matthias Kettner und Friedrich Heubel ergänzen die Charter of Medical Professionalism um ihren moralphilosophischen Hintergrund. Sie werten so die Profession als Gemeinschaft der Professionellen auf und stärken ihre Rolle bei der Verteidigung professionellen Handelns gegen die allfälligen Deformierungskräfte.

\section{Literatur}

1. Heubel F, Kettner M, Manzeschke A (Hrsg) (2010) Die Privatisierung von Krankenhäusern - Ethische Perspektiven. VS Verlag für Sozialwissenschaften, Wiesbaden 